\title{
Broadband vibration energy harvesting based on cantilevered piezoelectric bi-stable composites
}

\author{
A. F. Arrieta, ${ }^{1, a)}$ T. Delpero, ${ }^{1}$ A. E. Bergamini, ${ }^{2}$ and P. Ermanni ${ }^{1}$ \\ ${ }^{1}$ Centre of Structure Technologies, ETH Zurich, Leonhardstrasse 27, Zurich CH-8092, Switzerland \\ ${ }^{2}$ Empa, Mechanics for Modelling and Simulation, Überlandstrasse 129, Dübendorf CH-8600, Switzerland
}

(Received 5 March 2013; accepted 15 April 2013; published online 1 May 2013)

\begin{abstract}
Bi-stable composites are considered for vibration based energy harvesting, thanks to the broadband nature of their dynamic response. In this letter, a cantilevered piezoelectric bi-stable composite concept is introduced for broadband energy harvesting. The proposed configuration allows for exploiting the large strains developed close to the clamped root, significantly enhancing the harvesting effectiveness in comparison to previous settings. A simple model is used for designing the dynamic response aiming to maximise broadband oscillations. Experimental results reveal wide bands of high power conversion. Additionally, a shunting circuit suitable for broadband conversion is employed, further increasing the effectiveness of the proposed concept. (C) 2013 AIP Publishing LLC. [http://dx.doi.org/10.1063/1.4803918]
\end{abstract}

Conventional vibration energy harvesting devices have been designed to operate optimally at or very close to resonance. ${ }^{1}$ However, ambient vibrations generally show multiple frequencies which drift over time, rendering typical linear harvesters unsuitable for most practical applications. The idea of using nonlinearity for obtaining broadband energy conversion has been shown to be a viable solution to address this design bottleneck. ${ }^{2,3}$ Systems exhibiting bistability are a promising alternative for broadband energy harvesting owing to their nonlinear dynamic response. Amongst such systems, bi-stable composites which exhibit two statically stable shapes, or states shown in Fig. 1, feature additional advantages. ${ }^{5}$ First unlike beam-like structures, ${ }^{4}$ the two-dimensional plate-like behaviour allows for obtaining two different sets, one for each stable state, of linear and nonlinear modal resonances around which broadband dynamics appear. ${ }^{6}$ Second, these composites owe their bi-stability to purely elastic phenomena; hence, no external devices such as magnets are required to obtain the desired dynamics. Third, bi-stable composites easily integrate with piezoelectric materials yielding simple and reliable devices for operation. In this letter, a concept using cantilever piezoelectric bi-stable composites for broadband energy harvesting is proposed. The improved design featuring a symmetrical-unsymmetrical layup, ${ }^{8}$ presented in Fig. 2, is used for enabling the clamping of one edge of bi-stable composites allowing for exploiting high strains developed close to the root. The resulting cantilevered configurations, shown in Fig. 2, allow for improved energy conversion compared to previous designs where the bi-stable laminates could only be clamped from a small region ${ }^{7}$ limiting the available strains.

The broadband characteristics of bi-stable composites appear due to cross-well oscillation triggered by an external vibrations source. These types of oscillations arise with minimum actuation effort around the bending modes and nonlinear resonances, which are in general different for each stable state. ${ }^{9}$ The ability to place the modal frequencies for each

${ }^{\text {a)} E l e c t r o n i c ~ m a i l: ~ a n d r e s a r @ e t h z . c h ~}$ state is of chief importance for the resulting broadband oscillations. In particular, the separation between the first bending modes associated to the stable state, controls the range for which large amplitude oscillations are easily triggered. A larger separation between modes results in a wider range of cross-well oscillations, albeit at the potential cost of larger external vibration amplitude, as far from resonance the necessary effort to force snap-through increases. Hence, a design compromise reconciling a broader range of cross-well oscillations while maintaining desired low levels of external input must be reached. The dynamic model presented in Ref. 10 is used to achieve a suitable separation between modes whilst limiting the input amplitude for triggering cross-well oscillations. Dynamic response design parameters include lay-up, stacking sequence, and dimensions such as thickness and width. In this study the length from the clamped edge, $L_{x}$ in Fig. 2, is chosen as the design parameter to show the general procedure to obtain desired modal characteristics. The response is experimentally explored using the set-up shown in Fig. 3 to obtain frequency response functions (FRFs), presented in Fig. 4, for two different cases: specimen A and specimen B having $L_{x}=168 \mathrm{~mm}$ and $L_{x}=178 \mathrm{~mm}$, respectively. It is clear that the separation between the first bending modes for specimen $\mathrm{A}$ is larger than for specimen $\mathrm{B}$. Additionally, specimen B shows higher compliance suggesting less external actuation is required for triggering crosswell oscillations. The theoretical and experimental values for the first bending modal frequency for stable states 1 and 2 are compared in Table I, showing good agreement featuring errors ranging between $3 \%$ and $5 \%$.

The nonlinear behaviour of the bi-stable specimens is studied using experimental frequency response diagrams, which combine the concepts of FRFs and bifurcation diagrams to provide information of the dynamics response of a structure. ${ }^{5}$ These diagrams are obtained sweeping through a range of frequencies exciting the specimens with a single harmonic input for different base acceleration amplitudes. The response is sampled over several consecutive forcing periods of steady state motions and plotted using the forcing frequency as 


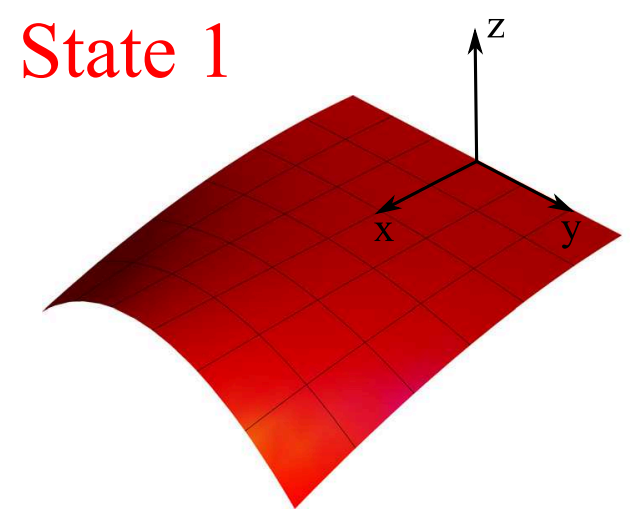

\section{State 2}

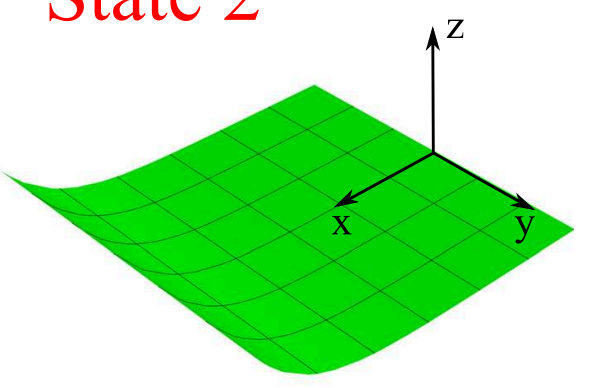

FIG. 1. Stable shapes of cantilevered bistable composites.

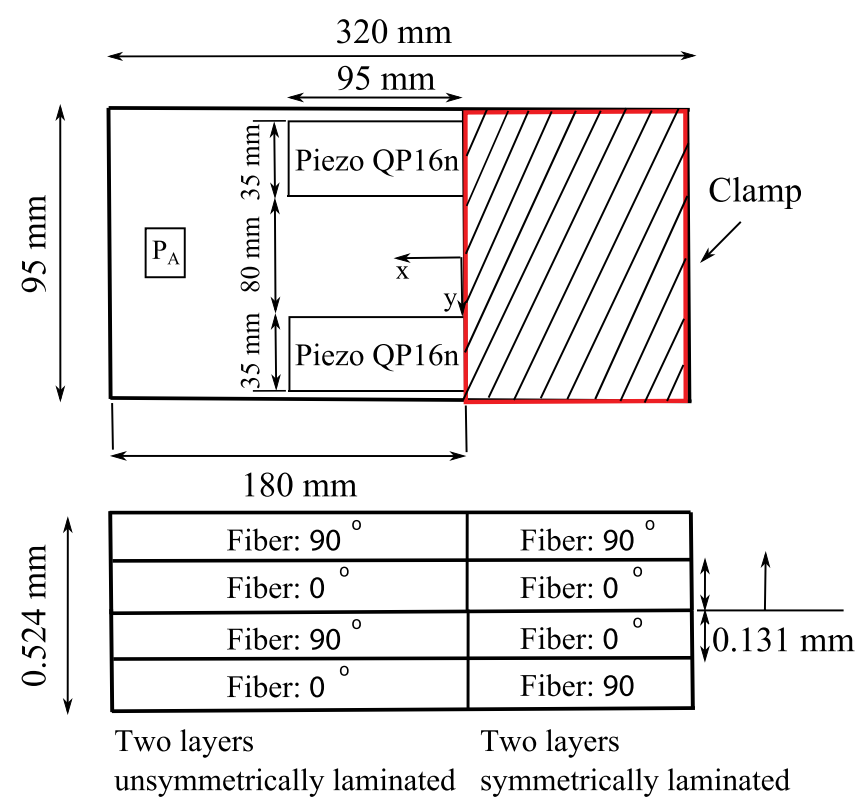

FIG. 2. Symmetric-unsymmetric lay-up enabling cantilever configuration and positioning of piezoelectric transducers.

parameter. When the response is linear, a single amplitude value is sampled for consecutive periods for a given forcing frequency. Conversely, several amplitude values for a given frequency indicate the presence of multiple harmonics in the response signalling nonlinear oscillations. In particular, a dense cluster of points covering several amplitudes for a forcing frequency evidences the appearance cross-well oscillations. The parameter space in terms of input frequency for the appearance of cross-well oscillations of the studied specimens is thus obtained with such diagrams. The cross-well oscillations

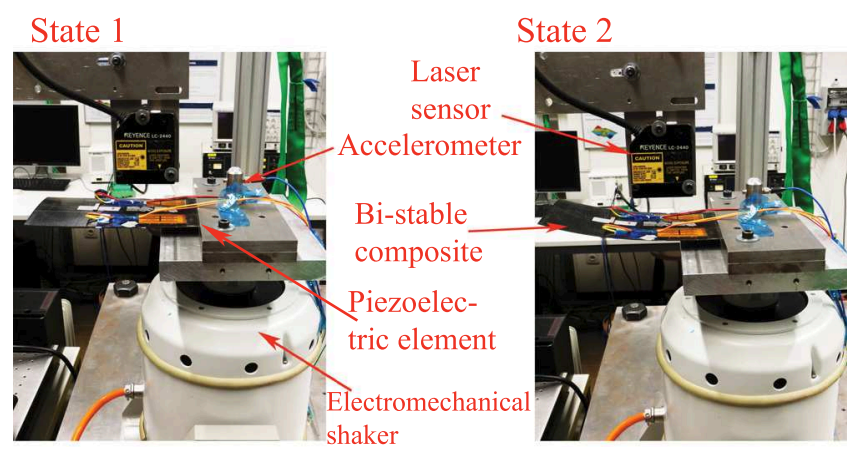

FIG. 3. Stable states of studied specimen and experimental set-up. frequency range exhibited by the tested specimens is only marginally affected by the direction of the sweep input used for studying the parameter space, showing the robustness of the concept to vibration excitation variations. Furthermore, tests for which the forcing amplitude is rapidly increased, simulating expected realistic vibration inputs, result in triggering the desired chaotic dynamics for the same range of frequencies as with frequency sweeps.

Cross-well oscillations appear first in the response of both specimens for a base acceleration level close to $0.1 \mathrm{~g}$, however, for a very limited frequency range. For specimen A, two regions of cross-well oscillations located about the first bending modal frequencies are obtained for an input amplitude of $0.33 \mathrm{~g}$, as shown in Fig. 5. These regions are disconnected as for this input amplitude deflections in the inter-resonance region fail to trigger snap-through. Nevertheless, for a higher level of excitation cross-well dynamics can be triggered yielding the complete range indicated by the dashed arrow. A different design compromise is shown for specimen B $\left(L_{x}=178 \mathrm{~mm}\right)$, for which a continuous wide range of cross-well oscillations is obtained for an input amplitude of $0.25 \mathrm{~g}$, as seen in Fig. 6. Comparing the broadband response of both specimens, cross-well dynamics appear for a range of $4.5 \mathrm{~Hz}$ for specimen $\mathrm{B}$, while two regions of 0.8 and $3.8 \mathrm{~Hz}$, respectively, are obtained for specimen A. The $42 \%$ lower level of forcing amplitude required

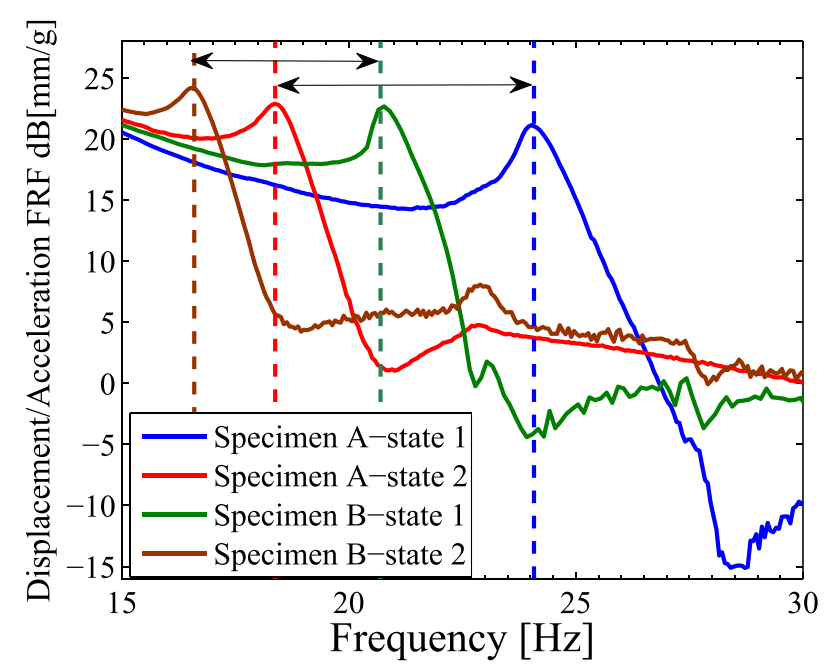

FIG. 4. Experimental displacement to base acceleration FRF of the studied specimens. Input amplitude $0.05 \mathrm{~g}$, below snap-through amplitude for all measurements. 
TABLE I. Experimental and theoretical comparison of first bending modes of each stable state for specimens A and B.

\begin{tabular}{lcccc}
\hline \hline \multirow{2}{*}{ Specimen } & \multicolumn{4}{c}{ Frequencies[Hz] } \\
\cline { 2 - 5 } & \multicolumn{2}{c}{ Experimental } & \multicolumn{2}{c}{ Simulated } \\
\hline \multirow{3}{*}{ A } & State 1 & State 2 & State 1 & State 2 \\
B & 24.1 & 18.4 & 25.2 & 19.1 \\
\hline \hline
\end{tabular}

to obtain cross-well dynamics for specimen B renders it better suited for the purposes of energy harvesting. Therefore, the characteristics of specimen B are studied in the following.

A resistor sweep is conducted to investigate the harvesting characteristics of the chosen design (i.e., specimen B) for distinct types of observed cross-well oscillations, namely, intermittency, fast chaos, and slow chaos. The difference between these cross-well oscillations is given by the qualitative nature of the time and spectral response associated to each type of behaviour. Intermittency shows fast bursts of high frequency snap-through, whereas the difference between slow and fast chaos is given by the fact that higher frequency components are clearly excited resulting in larger amplitude higher harmonics being observed in the spectral response of the latter. ${ }^{11}$ A single optimum resistance value yielding average root mean square (RMS) power maxima ranging from 3 to $5 \mathrm{~mW}$ for the studied behaviours is found, as seen in Fig. 7. The obtained levels of power with a simple resistor are relatively high when compared to other bi-stable systems given the low level of acceleration input $(0.25 \mathrm{~g})$.

Due the multi-harmonic nature of the cross-well dynamics, a suitable harvesting circuit capable of efficiently extract energy from the broad spectra oscillations featured by bi-stable composites is implemented. This is achieved by introducing the Synchronized Switching Harvesting on Inductor (SSHI) circuit based on the work presented in Ref. 12 and schematically

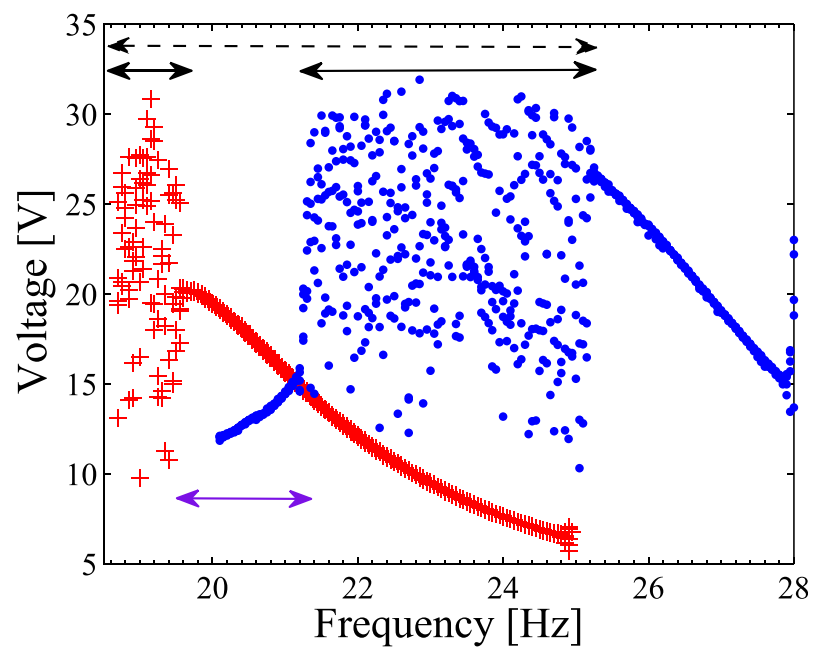

FIG. 5. Frequency response diagram for the voltage amplitude against forcing frequency of specimen A $\left(L_{x}=168 \mathrm{~mm}\right)$. Base acceleration level of $0.33 \mathrm{~g}$, and load resistance of $66 \mathrm{k} \Omega$. Blue dots and red crosses show frequency sweeps with initial condition on state 1 and 2, respectively. Purple arrow shows inter-resonance region of no cross-well dynamics. Dashed arrow shows potential range of cross-well dynamics.

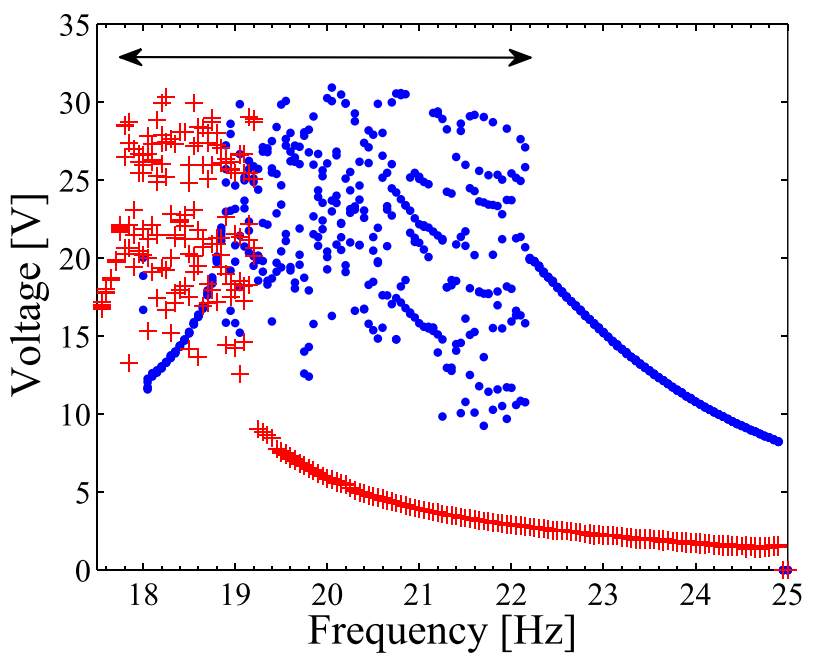

FIG. 6. Frequency response diagram for the voltage amplitude against forcing frequency of specimen B $\left(L_{x}=178 \mathrm{~mm}\right)$. Base acceleration level of $0.25 \mathrm{~g}$, and load resistance of $66 \mathrm{k} \Omega$. Blue dots and red crosses show frequency sweeps with initial condition on state 1 and 2, respectively. Arrows show regions of cross-well dynamics.

shown in Fig. 8. Similarly as before, a resistor sweep shown in Fig. 9 is conducted for the SSHI circuit revealing the maximum power is obtained for the same resistance value as in the simple resistive load case. However, the obtained power for the studied dynamic behaviours with the SSHI ranges from 35 to $55 \mathrm{~mW}$, showing a significant enhancement of the harvesting capabilities with an increase of an order of magnitude compared to the simple resistive load case. Furthermore, the obtained results are made more significant for this case as only one piezoelectric element is converting energy while the other is providing the small power required by the circuit. It is worth mentioning that the power required by the circuit is far lower than that produced by a piezoelectric element of the used size, implying that a much smaller transducer could be used in an optimised configuration further increasing the performance.

The proposed cantilevered configuration for piezoelectric bi-stable composites is demonstrated to offer significant performance enhancements yielding broadband high power energy harvesting by exploiting the large strains developed

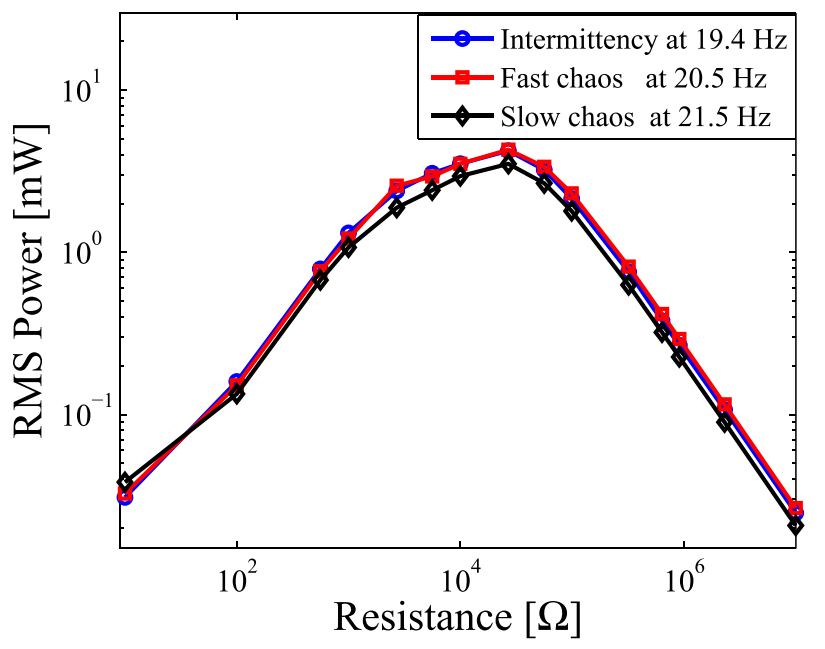

FIG. 7. Experimental average RMS power against load resistance for specimen B $\left(L_{x}=178 \mathrm{~mm}\right)$ : intermittency oscillations at $19.5 \mathrm{~Hz}$, fast chaotic oscillations at $20.5 \mathrm{~Hz}$, and slow chaotic oscillations at $21.5 \mathrm{~Hz}$. 


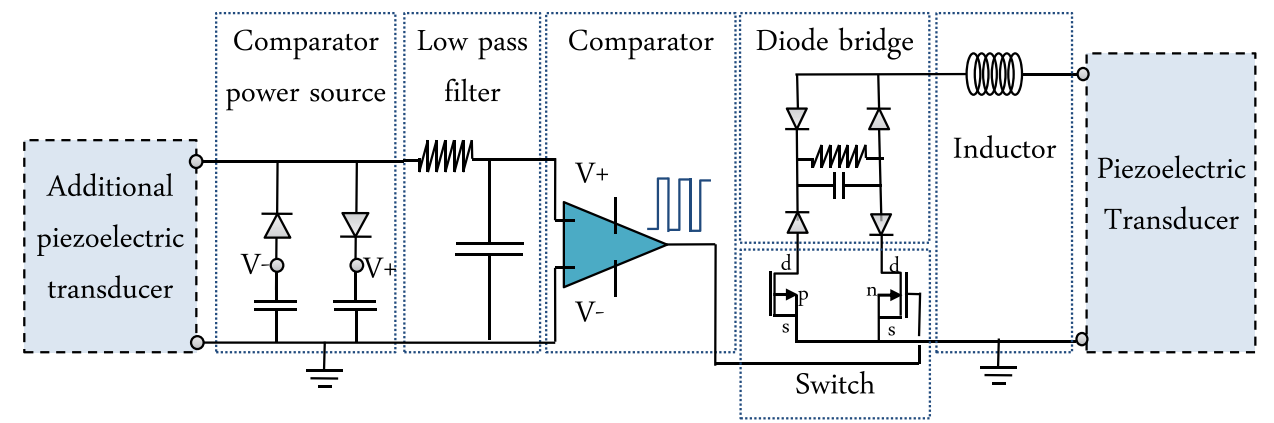

FIG. 8. SSHI circuit used for broadband conversion.

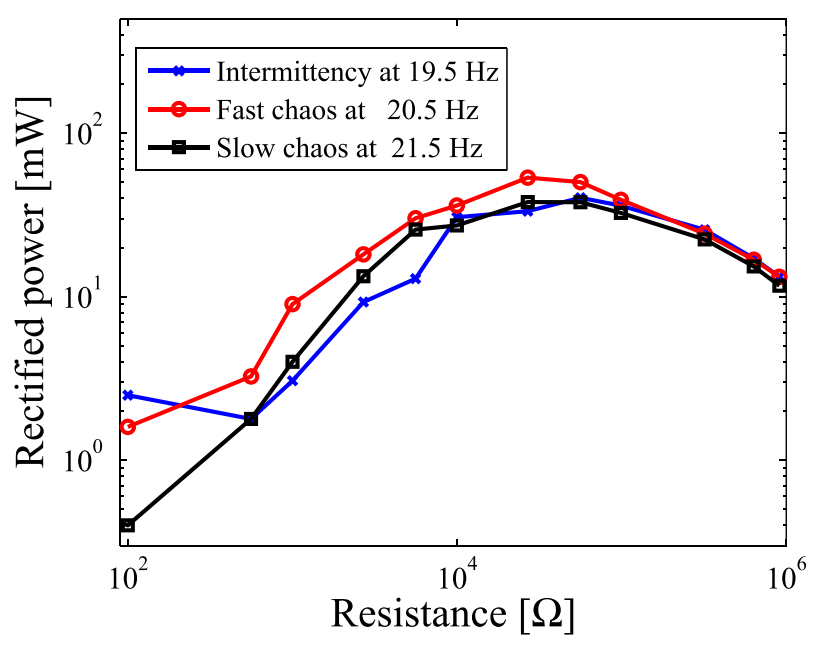

FIG. 9. Experimental rectified power against load resistance of the SSHI circuit for specimen B $\left(L_{x}=178 \mathrm{~mm}\right)$ : intermittency oscillations at $19.5 \mathrm{~Hz}$, fast chaotic oscillations at $20.5 \mathrm{~Hz}$, and slow chaotic oscillations at $21.5 \mathrm{~Hz}$.

close to the clamped root in combination with the wide spectra conversion capabilities of an SSHI circuit. The dynamic response is designed focusing on the separation between modal frequencies to obtain desired compromises of broadband dynamics and low amplitude input levels. Additional parameters for resonance placement and compliance tailoring, such as fiber orientation, can be designed offering expanded possibilities for multiple frequency regions of broadband high power energy harvesting.

The authors would like to thank the support of the ETH Research Commission and the Marie Curie Actions Co-fund
Program. Dr. A. F. Arrieta is funded through an ETH Postdoctoral Fellowship.

${ }^{1}$ Energy Harvesting Technologies, edited by S. Priya and D. J. Inman (Springer, 2009).

${ }^{2}$ L. Gammaitoni, I. Neri, and H. Vocca, "Nonlinear oscillators for vibration energy harvesting," Appl. Phys. Lett. 94, 164102 (2009).

${ }^{3}$ L. Tang, Y. Yang, and C. K. Soh, "Toward broadband vibration-based energy harvesting," J. Intell. Mater. Syst. Struct. 21, 1867-1897 (2010).

${ }^{4}$ D. A. W. Barton, S. G. Burrow, and L. R. Clare, "Energy harvesting from vibrations with a nonlinear oscillator," J. Vibr. Acoust. 132, 021009 (2010).

${ }^{5}$ A. F. Arrieta, P. Hagedorn, A. Erturk, and D. J. Inman, "A piezoelectric bi-stable plate for nonlinear broadband energy harvesting," Appl. Phys. Lett. 97, 104102 (2010).

${ }^{6}$ A. F. Arrieta, D. J. Wagg, and S. A. Neild, "Dynamic snap-through for morphing of bi-stable composite plates," J. Intell. Mater. Syst. Struct. 22, 103-112 (2011).

${ }^{7}$ A. F. Arrieta, A. Erturk, P. Hagedorn, and D. J. Inman, "Electromechanical modelling and experiments of a bistable plate for nonlinear energy harvesting," in ASME 2010 Conference on Smart Structures, Adaptive Structures and Intelligent Systems (SMASIS2010), Philadelphia, Pennsylvania, USA, September 28-October 12010.

${ }^{8} \mathrm{~F}$. Mattioni, "Thermally induced multi-stable composites for morphing aircraft applications," Ph.D. dissertation (University of Bristol, 2009).

${ }^{9}$ A. F. Arrieta, O. Bilgen, M. I. Friswell, and P. Hagedorn, "Dynamic control for morphing of bi-stable composites," J. Intell. Mater. Syst. Struct. 24, 266-273 (2012).

${ }^{10}$ A. F. Arrieta, O. Bilgen, M. I. Friswell, and P. Hagedorn, "Modelling and configuration control of multi-stable piezoelectric composites," (unpublished).

${ }^{11}$ See supplementary material at http://dx.doi.org/10.1063/1.4803918 for a description of the difference in spectral response of slow and fast chaotic oscillations.

${ }^{12}$ T. Delpero, L. Di Lillo, A. E. Bergamini, and P. Ermanni, "Energy harvesting module for the improvement of the damping performance of autonomous synchronized switching on inductance," J. Intell. Mater. Syst. Struct. 24(7), 837-845 (2013). 